\title{
Seasonal haze: Knowledge gaps and risk perception behaviours
}

\author{
Jing Han $\underline{\mathrm{Ng}}{ }^{1}{ }_{M B B S}$, Eng King $\underline{\operatorname{Tan}}{ }^{1,2}{ }_{M B B S}$
}

The seasonal haze in Southeast Asia has been a recurrent concern whenever we enter the southwest monsoon season (June-September). This phenomenon, caused by agricultural fires, has vast effects on multiple countries in the region. Besides extensive socioeconomic impact, the seasonal haze poses a significant public health threat, as numerous studies had demonstrated harmful effects of air pollution and haze on human health. Epidemiological studies conducted in various countries consistently demonstrate an association between haze exposure and respiratory, cardiovascular, oncological and psychological morbidity. ${ }^{1}$ Despite regional efforts, the Southeast Asian seasonal haze remains a persistent problem. It is thus imperative for people in this region to learn and practise personal protection against the haze.

In this issue of the Annals, $\mathrm{Ng}$ et al. sought to evaluate the knowledge and perceived risk of the seasonal haze in Singapore and the factors that affected protective behaviours taken by people during these haze episodes. $^{2}$ The investigators performed a crosssectional study of approximately 700 individuals, who lived in Singapore during the 2013 Southeast Asian haze crisis that caused record levels of pollution in the region. They identified study participants by performing random sampling of households in a public residential district in Singapore, and obtained data to assess the knowledge, perceived risks and protective behaviours for haze. The analyses provided a few salient observations of the attitude and behaviour of people towards the seasonal haze.

Firstly, protective behaviour against the seasonal haze was positively related to the knowledge level and perceived risk of haze, consistent with the Health Belief Model, which states that health behaviours are influenced by the perceived susceptibility and severity of a health threat. ${ }^{3}$ A different study in Southeast Asia also showed that greater knowledge and concern for the negative impact of haze on human health resulted in a higher likelihood for people to adopt protective measures. ${ }^{4}$
Secondly, basic knowledge and awareness of the seasonal haze and its negative impact on health were largely present in Singapore. However, while respondents generally demonstrated a basic understanding of the seasonal haze, crucial gaps in knowledge still existed. For example, a quarter of the study participants did not know that the Pollutant Standards Index (PSI) was used to measure haze severity and almost half thought that surgical masks would confer them protection against the haze. ${ }^{5}$ This represents a significant knowledge gap despite public education efforts.

Thirdly, factors associated with knowledge and risk perception of the seasonal haze were identified in the survey by $\mathrm{Ng}$ et al. A recent study found that a higher education background and monthly household income was associated with better knowledge and protective behaviours. ${ }^{6}$ Similarly, $\mathrm{Ng}$ et al. found that a lower education level and a habit of smoking were associated with poorer knowledge of the seasonal haze. This finding helps to highlight potential target groups for public health and haze education campaigns.

$\mathrm{Ng}$ et al. highlighted knowledge gaps of the seasonal haze within the Singapore population and drew attention to the frequently understated value of public education. Promoting protective behaviour by raising knowledge and risk perceptions of the seasonal haze can facilitate efforts to manage the consequences of the haze. It is interesting that higher education levels were linked to lower risk perception despite better knowledge of the haze situation. Higher monthly household income was also linked to fewer protective behaviours against the seasonal haze. This observation demonstrates the complex nature of human attitude and behaviour towards the haze, and highlights the need for a multidisciplinary and multifaceted approach to address some of the perceptions and behavioural responses. More studies are needed to further evaluate the relation between knowledge and perceived risk of haze, and the process for translation of awareness and knowledge into actual protective behaviours.

\footnotetext{
${ }^{1}$ National Neuroscience Institute, Singapore

${ }^{2}$ Duke-NUS Medical School, Singapore

Correspondence: Dr Eng King Tan, Department of Neurology, National Neuroscience Institute, Singapore General Hospital campus, Outram Road, Singapore 169608.

Email: gnrtek@sgh.com.sg
} 
Several challenges and limitations exist in the translation of knowledge and perceived risk to actual protective behaviour against the seasonal haze. While the general public may be equipped with the knowledge that surgical masks are not entirely effective against the haze, the widespread use of N95 masks may not always be feasible. Elderly, young children, people with cardiorespiratory comorbidities, and pregnant women with reduced lung volumes may not be able to tolerate the use of N95 masks. ${ }^{5}$ Yet, these are the individuals who are more vulnerable to the negative health effects of the haze. Likewise, those working in jobs with outdoor exposure may not have the luxury of choosing to stay indoors, despite knowledge and awareness of the importance in staying indoors during haze episodes. Clearly, an increase in knowledge and awareness of the haze alone is insufficient to promote individual protective behaviour. Government policies and organisational regulations can help to assist and facilitate personal protective behaviours. One example is the Workplace Safety and Health Act in Singapore that mandates employers to protect their employees' safety at work. On this premise, the Ministry of Manpower requires employers to identify vulnerable employees, reduce outdoor work, and provide employees who need to work outdoors with the appropriate N95 masks in situations of haze. ${ }^{7}$ Some of these measures may result in work and business disruptions for employers and should be adequately supported to avoid forming barriers for employers trying to protect employee health. Employer education and grants may help employers develop contingency plans and working arrangements such that their core operations or the relevant workplace safety measures are not affected by the haze.

It is also noteworthy that evidence for protective behaviours against the haze remains limited. Of the 7 protective behaviours listed by $\mathrm{Ng}$ et al., only 4 are backed by evidence. The use of masks, portable air cleaners and limitation of outdoor physical activities had Grade $\mathrm{C}$ evidence, while the monitoring of air pollution levels had Grade D evidence, based on grading used by the Global Initiative for Asthma (GINA). ${ }^{8}$ Further medical and scientific studies are required to validate protective behaviours and measures so that public education efforts may focus on the measures with proven effectiveness and robust evidence.
In addition, some lessons and observations from the current COVID-19 pandemic may be applicable to future haze crises. While the use of social media may facilitate quick dissemination of haze-related news, public health authorities need to manage misinformation and intervene swiftly when inaccurate or misleading information on the haze is disseminated. Mobile applications may help to provide real-time updates, alerts and advisories on the haze situation. The advancement and widespread use of telecommunication and videoconferencing platforms also provide a viable option for work, study and recreation during serious haze crises where physical and outdoor travel is not encouraged.

While the public health focus remains on COVID-19 in the current global pandemic, we should not lose sight of concurrent health threats. The Southeast Asian seasonal haze continues to be an important public health issue that should be studied and dealt with comprehensively.

\section{Acknowledgment}

EK Tan's work is supported by the National Medical Research Council.

\section{REFERENCES}

1. Ramakreshnan L, Aghamohammadi N, Fong CS, et al. Haze and health impacts in ASEAN countries: a systematic review. Environ Sci Pollut Res Int 2018;25:2096-111.

2. Ng KYY, Yeung W, Sou KL, et al. Factors influencing protective behaviours during haze episodes in Singapore: A population-based study. Ann Acad Med Singap 2021;50:514-26.

3. Rosenstock IM, Strecher VJ, Becker MH. Social Learning Theory and the Health Belief Model. Health Edu Q 1988;15:175-83.

4. De Pretto L, Acreman S, Ashfold MJ, et al. The Link between Knowledge, Attitudes and Practices in Relation to Atmospheric Haze Pollution in Peninsular Malaysia. PLoS One 2015;10: e0143655

5. Ministry of Health. FAQs on Haze Health Advisory, 2021. Available at: https://www.moh.gov.sg/resources-statistics/educationalresources/haze/faqs-on-haze-health-advisory. Accessed on 10 June 2021.

6. Low BS, Selvaraja KG, Ong TH, et al. Education background and monthly household income are factors affecting the knowledge, awareness and practice on haze pollution among Malaysians. Environ Sci Pollut Res Int 2020;27:30419-25.

7. Ministry of Manpower. Guidelines for employers on protecting employees from the effect of haze, 2020. Available at: https://www. mom.gov.sg/haze/guidelines-on-protecting-employees-from-haze. Accessed on 10 June 2021.

8. Carlsten C, Salvi S, Wong GWK, et al. Personal strategies to minimise effects of air pollution on respiratory health: Advice for providers, patients and the public. Eur Respir J 2020;55:1902056. 